\title{
Mucin-producing urothelial-type adenocarcinoma of prostate: report of two cases of a rare and diagnostically challenging entity
}

\author{
Michael W Curtis ${ }^{1}$, Andrew J Evans ${ }^{1}$ and John R Srigley ${ }^{2}$ \\ ${ }^{1}$ Department of Pathology and Laboratory Medicine, University Health Network, University of Toronto, \\ Toronto, Ontario, Canada and ${ }^{2}$ The Credit Valley Hospital, Mississauga, Ontario, Canada
}

\begin{abstract}
The differential diagnosis of mucin-producing adenocarcinoma of the prostate includes conventional prostatic adenocarcinoma with mucin production, secondary adenocarcinoma usually of colorectal origin and, very rarely, urothelial-type adenocarcinoma arising from either the prostatic urethra or proximal ducts. Conventional prostatic adenocarcinoma with mucin production is readily identified by routine microscopy and immunohistochemistry. The distinction between secondary adenocarcinoma and urothelial-type adenocarcinoma, however, can present a significant diagnostic challenge. In addition, documented examples of the latter in the prostate are exceptionally rare. A transurethral resection of prostate specimen and prostatic needle biopsies from two patients showing urothelial-type adenocarcinoma of the prostate were identified in our consultation files. One of the patients subsequently underwent a radical prostatectomy. Both patients had negative gastrointestinal endoscopic workups. Transurethral resection of prostate material from two patients with clinically confirmed secondary adenocarcinoma of colonic origin involving the prostate and a prostatectomy specimen with mucinous conventional prostatic adenocarcinoma were also identified for comparison purposes. Formalin-fixed, paraffin-embedded sections were stained for prostate-specific antigen (PSA), prostatic acid phosphatase, carcinoembryonic antigen, cytokeratin 7, cytokeratin 20 and high molecular weight cytokeratin $34 \beta E 12$. The urothelial-type adenocarcinoma cases were diffusely positive for cytokeratin 7 and focally positive for $34 \beta \mathrm{E} 12$ and cytokeratin 20 , consistent with an origin from the urothelium of the prostatic urethra or proximal prostatic ducts. In contrast, the secondary adenocarcinoma of colonic origin cases were diffusely cytokeratin 20 positive and either negative or focally positive for cytokeratin 7 and negative for $34 \beta E 12$. The mucinous conventional prostatic adenocarcinoma was positive for PSA and prostatic acid phosphatase and negative for cytokeratin 7, cytokeratin 20 and 34ßE12. All tumors were positive for carcinoembryonic antigen.
\end{abstract}

Modern Pathology (2005) 18, 585-590. doi:10.1038/modpathol.3800317

Keywords: urothelial carcinoma; mucinous adenocarcinoma; urethra; prostate; immunohistochemistry

Carcinoma arising in the male urethra, with or without involvement of the prostate, is uncommon and usually occurs during the sixth and seventh decades, with $95 \%$ of cases occurring after the age of 45 years. $^{1}$ Obstructive symptoms due to a urethral mass or stricture are the usual presenting features. ${ }^{2,3}$ Chronic irritation, infection, and trauma, including that from instrumentation used to relieve strictures,

Correspondence: Dr AJ Evans, MD, PhD, FRCPC, Department of Pathology and Laboratory Medicine, University Health Network, Princess Margaret Hospital, 610 University Avenue, Room 4-313, Toronto, Ontario, Canada M5G 2M9.

E-mail: andrew.evans@uhn.on.ca

Received 28 July 2004; revised and accepted 7 September 2004 are the major predisposing factors. ${ }^{1,2}$ In one series, $37 \%$ of patients had a history of venereal disease, $35 \%$ had stricture, and $7 \%$ had suffered trauma. ${ }^{3}$ It has been reported that up to $88 \%$ of patients with urethral carcinoma have a history of urethral stricture. The most common site for stricture, the bulbomembranous urethra, is also the most common location for carcinoma. The penile urethra is the next most common location, with the prostatic urethra being the least common site. ${ }^{4}$ Of 115 tumors reviewed by Melicow and Roberts ${ }^{1}$ and 34 cases reviewed by Ray, Canto, and Whitmore, ${ }^{2}$ only 18 cases and one case, respectively, were thought to have originated in the prostatic urethra. Most urethral carcinomas are transitional cell carcinomas 
and squamous cell carcinomas. Only 6\% are adenocarcinomas and these usually have glandular or papillary architecture. ${ }^{6}$ The presence of mucin pools, as described by Silverman et $a l^{5}$ and Loo and Chan, ${ }^{6}$ is extremely rare.

The differential diagnosis of mucin-producing adenocarcinoma found in the prostate most commonly includes conventional prostatic adenocarcinoma with mucin production and secondary adenocarcinoma, usually of colorectal origin, which has metastasized or directly extended into the prostate. Mucin-producing urothelial-type adenocarcinoma arising in the prostatic urethra or proximal large ducts of the prostate is also in the differential diagnosis. Although extremely rare, this entity can be a source of considerable diagnostic confusion when it appears in prostate needle biopsies or transurethral resection specimens. This is particularly true with respect to its distinction from secondary adenocarcinoma of colonic origin. These possibilities must be correctly distinguished for appropriate patient management.

Tran and Epstein ${ }^{7}$ reported two such cases of mucinous adenocarcinoma of urinary bladder type arising from the prostatic urethra that were confined to the prostate. Both tumors were characterized by pools of mucin and tall columnar epithelium reminiscent of that seen in colonic adenocarcinoma. Urethritis glandularis with colonic metaplasia and adenocarcinoma in situ were noted in the mucosa adjacent to the tumors. Immunohistochemically, both cases were negative for prostate-specific antigen (PSA) and prostatic acid phosphatase (PSAP), but positive for carcinoembryonic antigen (CEA).

We present two additional cases of mucin-producing urothelial-type adenocarcinoma that initially created considerable diagnostic confusion and describe an immunohistochemical panel that allowed the correct diagnosis to be made.

\section{Case reports}

\section{Case 1}

An 89-year-old man presented with urinary retention, bilateral flank pain, microscopic hematuria and renal insufficiency (attributed to bladder outlet obstruction) in December 2001. Digital rectal exam revealed a firm nodule in the right lobe of the prostate that, clinically, appeared to be prostate cancer with extracapsular spread. His serum PSA was $1.17 \mathrm{ng} / \mathrm{ml}$. Cystoscopy showed a friable outgrowth in the right side of the prostatic urethra. A transrectal ultrasound-guided biopsy of the prostate was performed which revealed a PSA-negative mucin-producing adenocarcinoma. A differential diagnosis that included secondary adenocarcinoma of gastrointestinal tract origin and a primary urethral adenocarcinoma was given. The patient then underwent endoscopic examination of his entire gastrointestinal tract that was remarkable only for two non-neoplastic colonic polyps. A metastatic workup was negative. Clinically, it was decided that radical surgery was not feasible owing to the patient's advanced age, medical comorbidities and the impression that the tumor could not be completely resected. Hormone treatment was not offered, as the tumor was PSA negative. External beam radiation therapy was considered to be the treatment of choice. Before the radiation therapy could commence, he underwent a transurethral resection of his prostate to relieve his obstructive symptoms. A significant amount of mucin could be seen coming from the prostate into the prostatic urethra during this procedure. Shortly after the transurethral resection, he became unwell and a CT scan showed liver metastases. He subsequently developed disseminated intravascular coagulation, presumed to be secondary to his mucin-producing tumor, and died 9 months after his initial presentation. No autopsy was performed.

\section{Case 2}

A 57-year-old man with a serum PSA of $0.3 \mathrm{ng} / \mathrm{ml}$ was found to have a nodule in the left lobe of his prostate in June 2001. He underwent a transrectal ultrasound-guided biopsy of the nodule that was reported as showing high-grade prostatic intraepithelial neoplasia. Eight months later, his serum PSA was $1.47 \mathrm{ng} / \mathrm{ml}$ and a second prostate biopsy was performed. This was reported as showing adenocarcinoma with mucin production, not otherwise specified. For reasons that are not clear from review of the patient's chart, a third prostate biopsy was performed in May 2002. This was also reported as showing mucinous adenocarcinoma, not otherwise specified. Prostatic and colorectal primary sites were suggested. Endoscopic evaluation of his gastrointestinal tract was negative. Slides from the three prostatic needle biopsies were sent to one of us in consultation (JRS) and a diagnosis of mucin-producing urothelial-type adenocarcinoma of the prostate was rendered. In August 2002, the patient underwent a radical prostatectomy with bilateral pelvic lymph node dissection and the patient has remained free of locally recurrent or metastatic disease through 16 months of follow-up.

\section{Materials and methods}

Formalin-fixed, paraffin-embedded tissue was available from the transurethral resection specimen from case \#1 and all three of the prostate needle biopsies as well as the radical prostatectomy specimen from case \#2. Formalin-fixed, paraffin-embedded tissue from transurethral resection specimens from two patients with clinically confirmed secondary adenocarcinoma of colonic origin involving the prostate, along with formalin-fixed, paraffin-embedded tissue from a prostatectomy specimen containing 
mucinous conventional adenocarcinoma of prostate were also retrieved from our files for comparison purposes. Sections $(5 \mu \mathrm{m})$ from all of these tumors were stained using primary antisera against PSA (1:3000, Dako Diagnostics), PSAP (1:3000, Dako), CEA (1:5000, Biomeda), cytokeratin 7 (CK7) (1:2000, Dako), cytokeratin 20 (CK20) (1:1000, Dako) and high molecular weight cytokeratin $34 \beta$ E12 (1:50, Dako) at the indicated dilutions using the Ventana automated immunostaining procedure. The intensity of immunohistochemical staining was evaluated semiquantitatively using the following system; ' +++ ' for strong, diffuse staining, ' ++ ' for moderate staining, ' + ' for weak and focal staining and '-' for negative staining.

\section{Results}

\section{Histopathology and Immunohistochemical Findings}

The transurethral resection of prostate from case \#1 consisted of $20 \mathrm{~g}$ of tissue fragments. Histologic sections showed detached fragments of mucinproducing adenocarcinoma with glandular and cribriform architecture infiltrating the prostatic parenchyma. The tumor cells were tall and columnar with marked nuclear atypia (Figure 1). Areas of 'dirty' necrosis were present. The hematoxylin and eosin morphology of this tumor was virtually identical to that of secondary adenocarcinoma of colonic origin involving the prostate (Figure 1). It also resembles conventional prostatic adenocarcinoma with mucin production at intermediate magnification (Figure 1). At higher magnification, however, one can generally recognize the bland, cuboidal epithelium typical of prostatic adenocarcinoma allowing its identification on hematoxylin and eosin morphology alone. Immunohistochemically, the tumor showed completely negative staining for PSA and PSAP. It was diffusely positive for CK7, CK20, CEA and focally positive for $34 \beta$ E12 (Table 1 and Figure 2). Taken together, the morphology and immunophenotype were those of mucin-producing urothelial-type adenocarcinoma involving the prostate.

All three prostate needle core biopsies from case \#2 were reviewed by one of us (JRS). The histology and immunophenotype were identical to the features described for case \#1. Figure 3 shows the H\&E appearance of mucin-producing urothelial-type adenocarcinoma on needle biopsy.

The prostatectomy specimen from case \#2 was submitted in toto for histologic examination. These slides showed a tumor measuring $2.5 \mathrm{~cm}$ in greatest dimension that was centered around, and bulged into, the prostatic urethra (Figure 4). There was no urethritis glandularis, intestinal metaplasia, adenocarcinoma in situ or intraurothelial neoplasia (of high or low grade) in sections showing uninvolved urethral mucosa adjacent to the tumor. The bulk of the tumor was located in the left apical-posterior
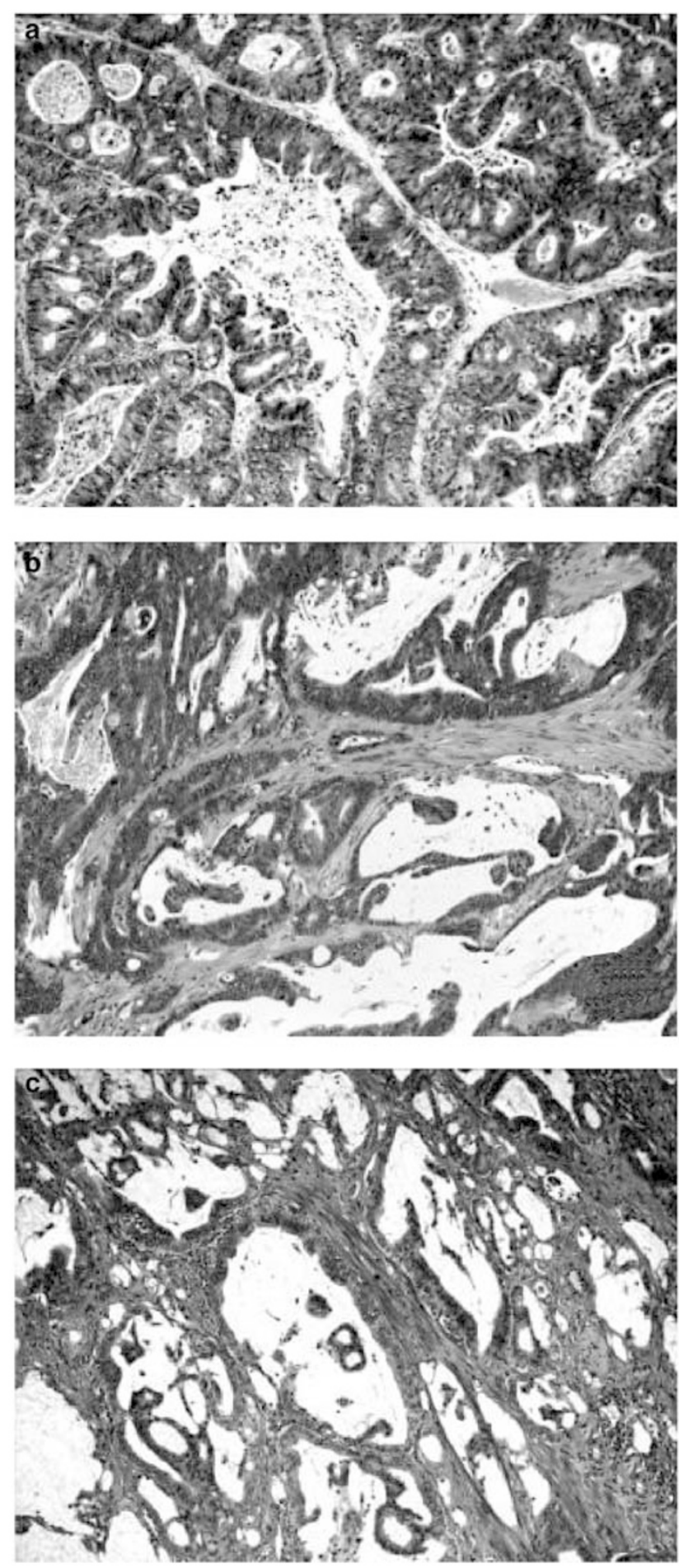

Figure 1 Microscopic features of the three main lesions comprising the differential diagnosis of mucin-producing adenocarcinoma involving the prostate: (a) urothelial-type adenocarcinoma as it appeared in the TURP specimen from case \#1; (b) colonic adenocarcinoma with secondary involvement as it appeared in a palliative TURP specimen; (c) conventional acinar-type prostatic adenocarcinoma with mucin production in a radical prostatectomy specimen (H\&E stain for all panels, $\times 100)$.

aspect of the gland and was characterized by prominent mucinous dissection of the prostatic stroma (Figure 4). In addition, there were numerous 
Table 1 Immunohistochemical staining patterns

\begin{tabular}{lccccc}
\hline & $P S A$ & PSAP & CK7 & CK20 & $34 \beta E 12$ \\
\hline CPA & +++ & +++ & - & - & - \\
UTA & - & - & +++ & + & ++ \\
SAC & - & - & - & ++ & - \\
\hline
\end{tabular}

Conventional prostatic adenocarcinoma with mucin production (CPA); urothelial-type adenocarcinoma arising in the prostatic urethra or proximal prostatic ducts (UTA); secondary adenocarcinoma of colonic origin involving the prostate (SAC); ' +++'=strong, diffuse staining; '++' = moderate staining; '+' = weak or focal staining; '-' = negative staining.
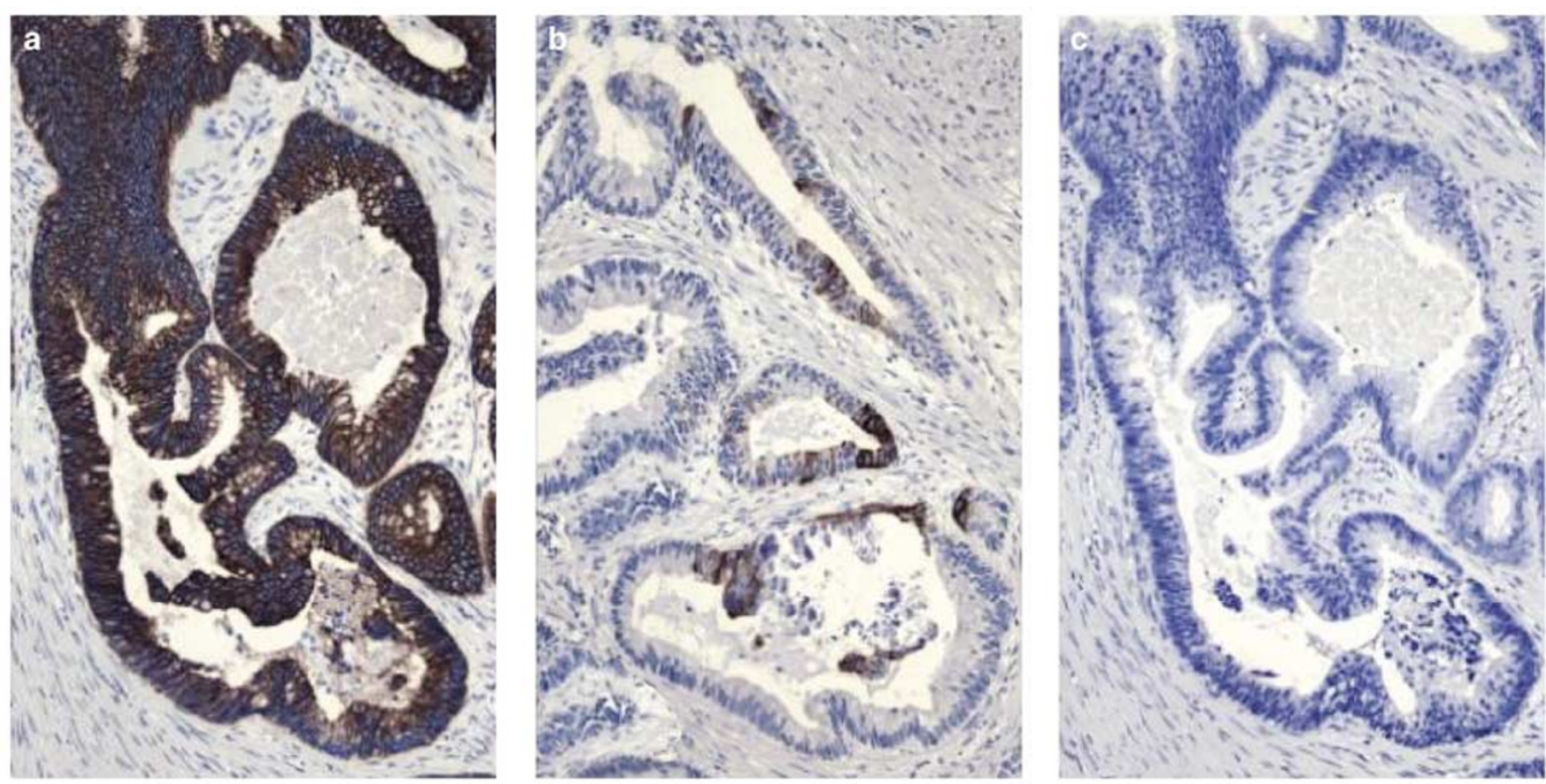

Figure 2 Immunohistochemical staining characteristics of mucin-producing urothelial-type adenocarcinoma of the prostate: (a) diffusely positive staining for CK7; (b) focally positive staining for 34 $\beta$ E12; (c) completely negative staining for PSA (all panels $\times 100$ ).

microscopic satellite foci in the peripheral zone of the left lobe (all $<2 \mathrm{~mm}$ in maximum dimension) of tumor showing intraductal spread within the prostate (Figure 4). The tumor extended to the left apical and peripheral margins of resection. The seminal vesicles and regional lymph nodes were negative for malignancy. Sections from the peripheral zone also showed high-grade prostatic intraepithelial neoplasia and two separate microscopic foci ( 1 and $2 \mathrm{~mm}$ in greatest dimension) of conventional acinar-type prostatic adenocarcinoma of Gleason score 6/10 (3+ 3 ) that were spatially separate from foci of urothelial-type adenocarcinoma (not shown).

Representative sections from cases of secondary adenocarcinoma of colonic origin and conventional prostatic adenocarcinoma with mucin production were used for comparison purposes with the two mucin-producing urothelial-type adenocarcinoma cases and the immunohistochemical staining patterns for all three tumor types are summarized in Table 1. Of note, the examples of secondary colonic adenocarcinoma showed the expected positive immunostaining with CK20 and CEA and were negative or only focally positive for CK7. Stains for $34 \beta \mathrm{E} 12$, PSA and PSAP were completely negative. The conventional prostatic adenocarcinoma with mucin production was positive for PSA, PSAP and CEA and was negative for CK7, CK20 and $34 \beta E 12$.

\section{Discussion}

Urothelial-type adenocarcinoma of prostate is a very rare, but important entity for pathologists to be aware of. These tumors can create considerable diagnostic confusion with respect to its distinction from secondary adenocarcinoma of colonic origin. The two cases presented herein demonstrate that is essentially impossible to distinguish urothelial-type adenocarcinoma of the prostate from secondary colonic adenocarcinoma based on H\&E morphology alone, especially when they appear on prostate needle biopsies and/or TURP specimens. In radical prostatectomy specimens, the diagnosis of 

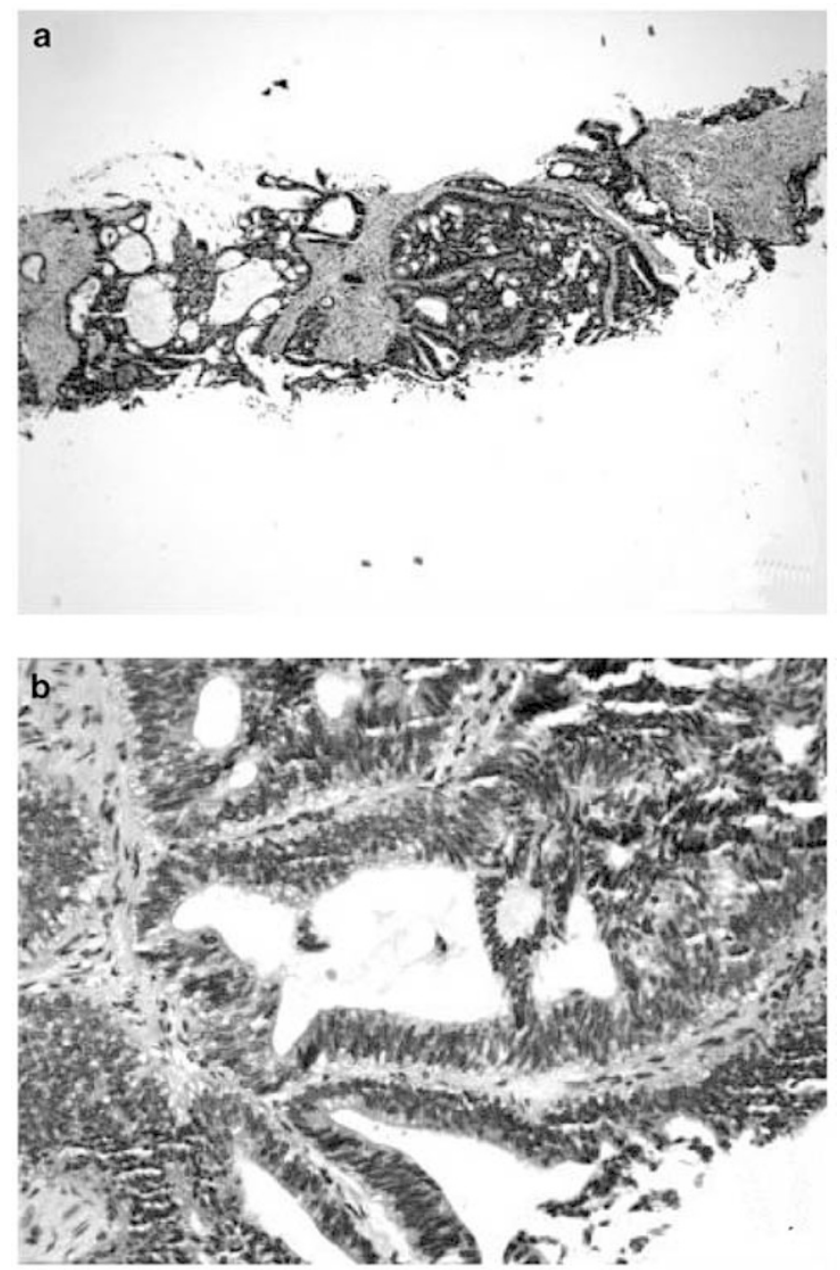

Figure 3 Microscopic features of mucin-producing urothelialtype adenocarcinoma of the prostate as it appeared in a needle core biopsy in case \#2 (H\&E stain, (a) $\times 25$ and (b) $\times 100)$.

urothelial-type adenocarcinoma arising in the prostatic urethra or proximal prostatic ducts may be more apparent, given the greater likelihood of finding precursor lesions such as glandular metaplasia of the urothelium, or urethritis glandularis, and adenocarcinoma in situ. ${ }^{7}$ As we have shown, an immunohistochemical panel that includes CK7, CK20, and $34 \beta$ E12 can be useful in establishing the correct diagnosis. Positive staining for CK7 and $34 \beta$ E12, with or without positive immunoreactivity for CK20, represents the immunophenotype expected for carcinomas of urothelial origin ${ }^{8}$ and supports the diagnosis of urothelial-type adenocarcinoma. Tamboli et $a l^{9}$ have shown that the addition of villin to such a panel is helpful in distinguishing between colonic adenocarcinoma metastatic to the urinary tract (villin positive) and primary urothelial carcinoma showing glandular differentiation (villin negative). Villin alone was not helpful in distinguishing between metastatic colonic adenocarcinoma and primary enteric-type adenocarcinoma of the urinary tract, as both entities show positive staining. a
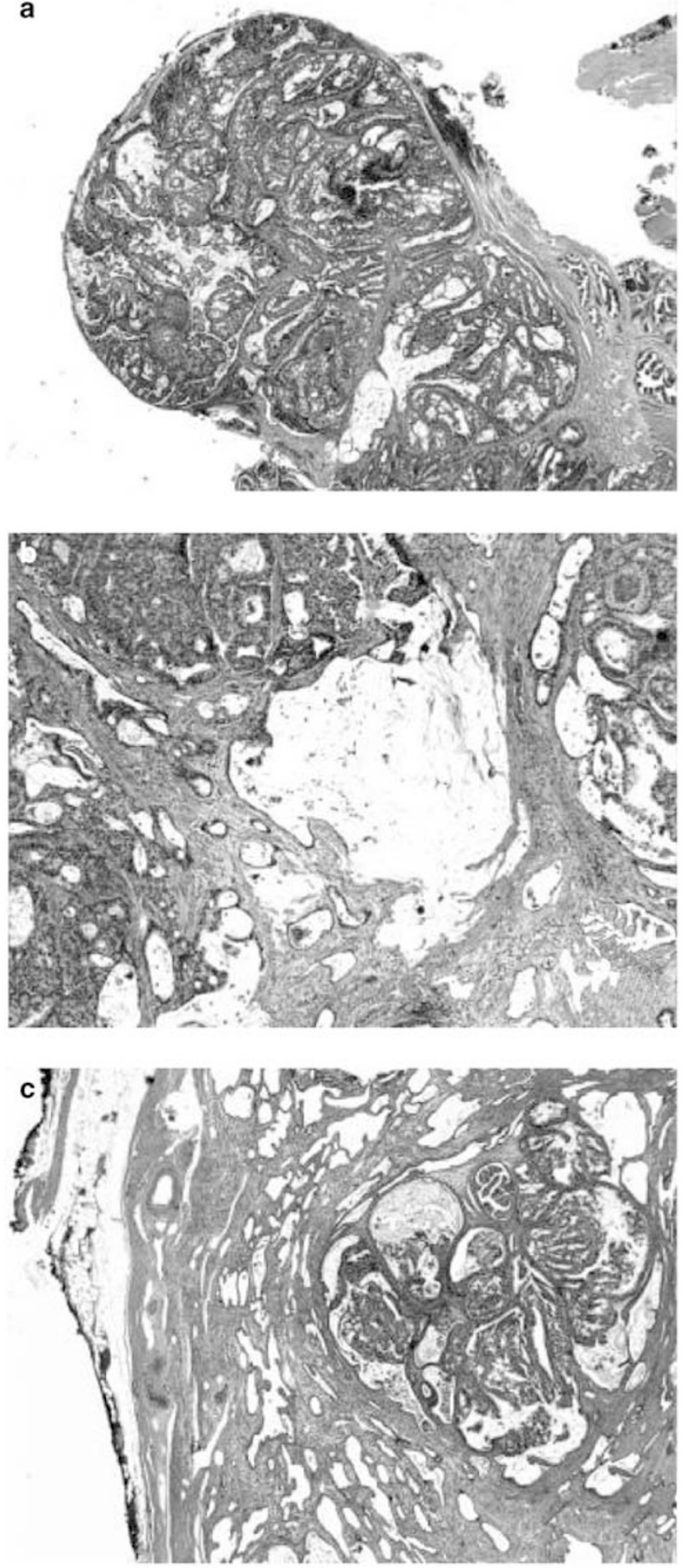

Figure 4 Microscopic features of the mucin-producing urothelialtype adenocarcinoma of prostate as it appeared in the radical prostatectomy specimen from case \#2: (a) exophytic tumor projecting into the prostatic urethra; (b) extensive mucinous dissection of the prostatic stroma; (c) section from the peripheral zone showing one of several microscopic satellite foci of intraductal spread with the prostate (H\&E stain, all panels $\times 50)$.

The two cases described in our report would be considered as primary enteric-type adenocarcinomas. As such, villin staining would not be expected 
to be of help in distinguishing urothelial-type adenocarcinoma from secondary colonic adenocarcinoma. Interestingly, Tamboli et al found that all four examples of primary adenocarcinoma of the urinary bladder they studied were negative for CK $7,{ }^{9}$ in contrast with our findings for urothelial-type adenocarcinoma of the prostate.

Establishing the correct diagnosis of urothelialtype adenocarcinoma involving the prostate is extremely important for several reasons. In both of our cases, the patients were subjected to extensive and invasive investigations to exclude the presence of a primary tumor in their gastrointestinal tracts. It may be possible to avoid these investigations, the discomfort and risks associated with them, and the potential delay in the initiation of treatment if the correct diagnosis had been established at the time of prostate biopsy or transurethral resection. In addition, establishing the correct diagnosis will prevent the potential initiation of inappropriate hormone therapy that might occur if the tumor were to be misconstrued as a variant of acinar-type prostatic adenocarcinoma.

In general, adenocarcinoma arising in the male urethra can originate from urethritis glandularis of the urothelium and/or squamous mucosa, especially when the metaplasia is of intestinal type. Other sources include periurethral structures such as Cowper's (bulbourethral) or Littre's glands. It is may be quite difficult to determine the source of these tumors as they may be diagnosed late in the course of the disease and after telltale precursor lesions have been obliterated by the growing mass.

With respect to urothelial-type adenocarcinoma involving the prostate, the report of Tran and Epstein $^{7}$ provided evidence that these tumors most likely arise from malignant transformation of urethritis glandularis involving the urothelium lining the prostatic urethra or proximal prostatic ducts, followed by the development of adenocarcinoma in situ and invasive adenocarcinoma.

The rarity of urothelial-type adenocarcinoma involving the prostate prevents making any conclusions with respect to clinical characteristics, behavior and treatment, other than to say that hormone therapy would be inappropriate. The two cases in our report bring the total number of cases in the literature to four, after Tran and Epstein ${ }^{7}$ described the first two cases in 1996. Their first patient was a 73-year-old man treated by radical prostatectomy who remained well without evidence of metastases through 1 year of follow-up. The second patient was a 68-year-old man treated by simple retropubic prostatectomy with recurrence of the tumor 4 years later that was treated with radiation therapy with a modest response. In our first patient, the tumor was more aggressive than the cases described by Tran and Epstein, culminating in death of the patient with liver metastases within 1 year of presentation. Our second patient has remained disease free for over 16 months of follow-up after radical prostatectomy, despite having microscopically positive resection margins.

In summary, we have described two additional cases of urothelial-type adenocarcinoma involving the prostate that have arisen in the prostatic urethra and/or proximal prostatic ducts. We have shown how an immunohistochemical panel that includes CK7, CK20, 34 $\beta$ E12, PSA and PSAP can be of value in establishing the correct diagnosis, especially distinguishing between urothelial-type adenocarcinoma and secondary adenocarcinoma of colonic origin involving the prostate. The documentation of more cases of urothelial-type adenocarcinoma involving the prostate is required to strengthen the contention that they arise in the prostatic urethra or proximal prostatic ducts, as well to determine the clinical behavior and establish optimal treatment.

\section{Acknowledgements}

We thank Dr D Zhang, Brandon, MB and Dr HR Wightman, Winnipeg, MB for contributing one case with clinical follow-up.

\section{References}

1 Melicow MM, Roberts TW. Pathology and natural history of urethral tumors in males. Review of 142 cases. Urology 1978;11:83-89.

2 Ray B, Canto AR, Whitmore WF. Experience with primary carcinoma of the male urethra. J Urol 1977; 117:591-593.

3 Grabstald H. Tumors of the urethra in men and women. Cancer 1973;32:1236-1253.

4 Kaplan GW, Bulkley GJ, Grayhack JT. Carcinoma of the male urethra. J Urol 1967;98:365-371.

5 Silverman ML, Eyre RC, Zinman LA, et al. Mixed mucinous and papillary adenocarcinoma involving the male urethra, probably originating in periurethral glands. Cancer 1981;47:1398-1402.

6 Loo KT, Chan JKC. Colloid adenocarcinoma of the urethra associated with mucosal in situ carcinoma. Arch Pathol Lab Med 1992;116:976-977.

7 Tran KP, Epstein JI. Mucinous adenocarcinoma of urinary bladder type arising from the prostatic urethra. Distinction from mucinous adenocarcinoma of the prostate. Am J Surg Pathol 1996;20:1346-1350.

8 Genega EM, Hutchinson B, Reuter VE, et al. Immunophenotype of high-grade prostatic adenocarcinoma and urothelial carcinoma. Mod Pathol 2000;13:1186-1191.

9 Tamboli P, Mohsin SK, Hailemariam S, et al. Colonic adenocarcinoma metastatic to the urinary tract versus primary tumors of the urinary tract with glandular differentiation. A report of 7 cases and investigation using a limited immunohistochemical panel. Arch Pathol Lab Med 2002;126:1057-1063. 